\title{
Drugs Related Changes of Haemoglobin and CD4 Counts in HIV-Infected Patients on Antiretroviral Therapy
}

\author{
Authors \\ Deebii, $\mathbf{N}^{1^{*}}$, Orluwene, $\mathbf{C G}^{2}$, Korubo, K. $\mathbf{I}^{1}$, and Njoku, $\mathbf{B}^{3}$ \\ ${ }^{1}$ Department of Haematology, Blood Transfusion and Immunology, University of Port Harcourt, \\ Rivers State, Nigeria \\ ${ }^{2}$ Department of Chemical Pathology, University of Port Harcourt, Rivers State, Nigeria \\ ${ }^{3}$ Department of Human Physiology, Faculty of Basic Medical Sciences, University of Port Harcourt, \\ Rivers State, Nigeria \\ *Corresponding Author

\section{Numbara Deebii} \\ Department of Haematology, Blood Transfusion and Immunology, University of Port Harcourt, \\ Rivers State, Nigeria \\ Email: numbarakomene@yahoo.com, +2347031214545
}

\begin{abstract}
The present study examines the effects of antiretroviral therapy (ART) on two basic immunohaematological parameters over time. We performed a retrospective study of HIV-infected treatment-nä̈ve patients starting a first line ART between June 2014 and January 2015, and followed-up for 12 weeks. On the whole, 254HIV-infected treatment naive patients were enrolled: 100 patients on tenoforvir (TDF) based regimen was compared with 102 and 52 non-TDF based regimen and treatment-nä̈ve patients respectively. Samples were collected at three different points (at baseline, 4 weeks and 12 weeks).CD4 and haemoglobin $(\mathrm{Hb})$ were found to be significantly higher $(p=0.001)$ in the TDF group as compared to non-TDF groups after 12 weeks of follow-up, although there was no significant difference between the Non-TDF group and ART-naive group at 4 and 12 weeks in both parameters. These results demonstrate that the initiation of a TDF based-regimen is followed by an increase in lymphocyte proliferation and lymphocyte-mediated cytotoxicity compared to other non-TDF based-regimen group. However we suggest that TDF should be part of an antiretroviral regimen in immunosuppressed patients at risk of anaemia.
\end{abstract}

Keywords: HIV, Tenoforvir (TDF), CD4 and immunosuppressed

\section{INTRODUCTION}

Several longitudinal studies have described infection with human immunedeficiency virus (HIV) causes a progressive dysfunction of the immune system in which there is reduced number of circulating CD4 $\mathrm{T}$ cells ${ }^{1}$ which is preceded by increased levels of circulating HIV RNA. This predicts disease progression, with development of acquired immune deficiency syndrome (AIDS) and death in the absence of therapy. The CD4 count is the most important laboratory indicator of immune function in HIV-infected patients. It also 
the strongest predictor of subsequent used to assess immunity, disease progression and survival according to findings from clinical trials and cohort studies $^{2}$. Thus CD4 is used to assess a patient's immunologic response to antiretroviral therapy. CD4 count response to ART varies widely but a poor CD4 response in patients with viral suppression is rarely an indication for modifying an ARV regimen.

Low Heamoglobin $(\mathrm{Hb})$ also characterizes the malfunction of the immune system in HIV infection and has been suggested as additional predictive markers of HIV disease progression. ${ }^{3}$ New treatment strategies, including the use of effective HIV-specific protease inhibitors such as tenofovir (TDF) or lavimidine, have swept the scientific community withhope caused by dramatic reductions of viral load and similar increases of CD4 cell counts. ${ }^{4}$. Similarly the use of protease inhibitors and triple therapy in clinical practice has been associated with a marked lowering of HIV-related morbidity. . However, clinical reports of severe opportunistic infections in the presence of satisfactory responses to antiretroviral treatment as evaluated by increases in CD4 cell counts ${ }^{6}$, have suggested that an increase in CD4 cell count may not always be accompanied by a similar augmentation of the functional capacity of the immune system, or that specific immune defects may persist despite treatment-induced reconstitution of other parts of the immune system.

\section{MATERIALS AND METHOD \\ Study population}

A longitudinal study of $254 \mathrm{HIV}$-infected naive patients was started between June 2014 and January 2015 at the HIV clinic of the University of Port Harcourt teaching hospital, a reference hospital for tropical infection and disease located in Port Harcourt, Nigeria. HIV-infected Naïve Patients never exposed to ART were invited to participate in the study so far they can be allocated in to any of the three groups; patients to be place on TDF regimen, patients to be place on Non-TDF regimen and HIV-infected patients that will not be exposed to antiretroviral therapy during the study period. From every patient's social-demographics details of age, sex, occupation, education, income, and HIV status (CD4 T-lymphocyte count) were recorded at the time of recruitment in to the study. Samples were collected at three times point (at baseline, after 4 weeks and after 12 weeks of follow-up). Periods of drug interruption for any reason, if occurring, were discounted. Treatment was provided without any cost to patients.

\section{Statistical analysis}

Continuous values are given as mean and standard deviation while categorical data are given as percentages. The three groups of patients analyzed were first compared using chi-squared for categorical data and pair tests for continuous variables. The level of significance was 0.05 . All statistical analyses were performed using SPSS version 20.0 software package (SPSS Inc., Chicago, Illinois, USA).

\section{RESULTS}

A total of two hundred and sixty seven patients were recruited in to the study from June 2014 to January 2015. Complete data were obtained from 254 patients while 13 patients were lost to followup. Of the 254 patients, 202 patients started a first line antiretroviral therapy (100 Patients on TDF and 102 patients on Non-TDF Antiretroviral therapy) and during the study period all patients met inclusion and exclusion criteria. Among these 202 enrolled patients, $100(39.37 \%)$ initiated tenofovir-containing regimen, $102 \quad(40.16 \%)$ initiated tenofovir-sparing regimens and 52 (20.47\%) antiretroviral Naïve patients. Baseline demographic characteristics and laboratory features were compared among the groups. 
Table 1: Age and Sex Characteristics of the study population

\begin{tabular}{lllll}
\hline & Mean Age & No. of patient & Std. Deviation & P-Value \\
\hline SEX & & & & \\
Male & 36.92 & $85(33.46 \%)$ & 7.69 & $\begin{array}{l}\text { (Z-test }) \\
\mathbf{p}=\mathbf{0 . 0 0 2}\end{array}$ \\
Female & 33.70 & $169(66.54 \%)$ & 7.97 & \\
Total & $\mathbf{3 4 . 7 7}$ & $\mathbf{2 5 4}(\mathbf{1 0 0 \%})$ & $\mathbf{8 . 0 1}$ & \\
ART Regimen group & & & & \\
TDF & 35.23 & $100(39.37 \%)$ & 7.68 & (ANOVA) \\
N-TDF & 35.49 & $102(40.16 \%)$ & 8.68 & $\mathbf{0 . 0 6 9}$ \\
D-NAIVE & 32.50 & $52(20.47 \%)$ & 6.93 & $\mathbf{8 . 0 2}$ \\
Total & $\mathbf{3 4 . 7 7}$ & $\mathbf{2 5 4}(\mathbf{1 0 0 \%})$ &
\end{tabular}

\section{Characteristic of patients}

The mean age of the male and female patients were; $36.92(33.46 \%)$ and, $33.70(66.54 \%)$ years respectively with a statistically significant difference of $\mathrm{P}=0.001$. The minimum and maximum age of the study population of the male and female patients were; 50:20, and 48:18 years respectively. Figure 1 shows that at baseline there was a statistically significant different between the CD4 of the treatment-Naïve group and the CD4 of the TDF and Non-TDF groups $(\mathrm{p}=0.000$ and 0.000 respectively). After 4 weeks of follow-up, there was an increase in the CD4 of the drug treatment groups but when compared with the treatment Naïve group, the different was highly statistically significant $(\mathrm{p}=0.000$ and 0.000 respectively) (Figure 2). After 12 weeks of exposure and follow there was a significant increase in the TDF group as compared with the treatment Naïve and Non-TDF groups. Although there was no statistically significant different between the TDF group and the Non-TDF group, a significant different was observed between the TDF and the treatment Naïve group $(\mathrm{p}=0.04)$ (Figure 3).

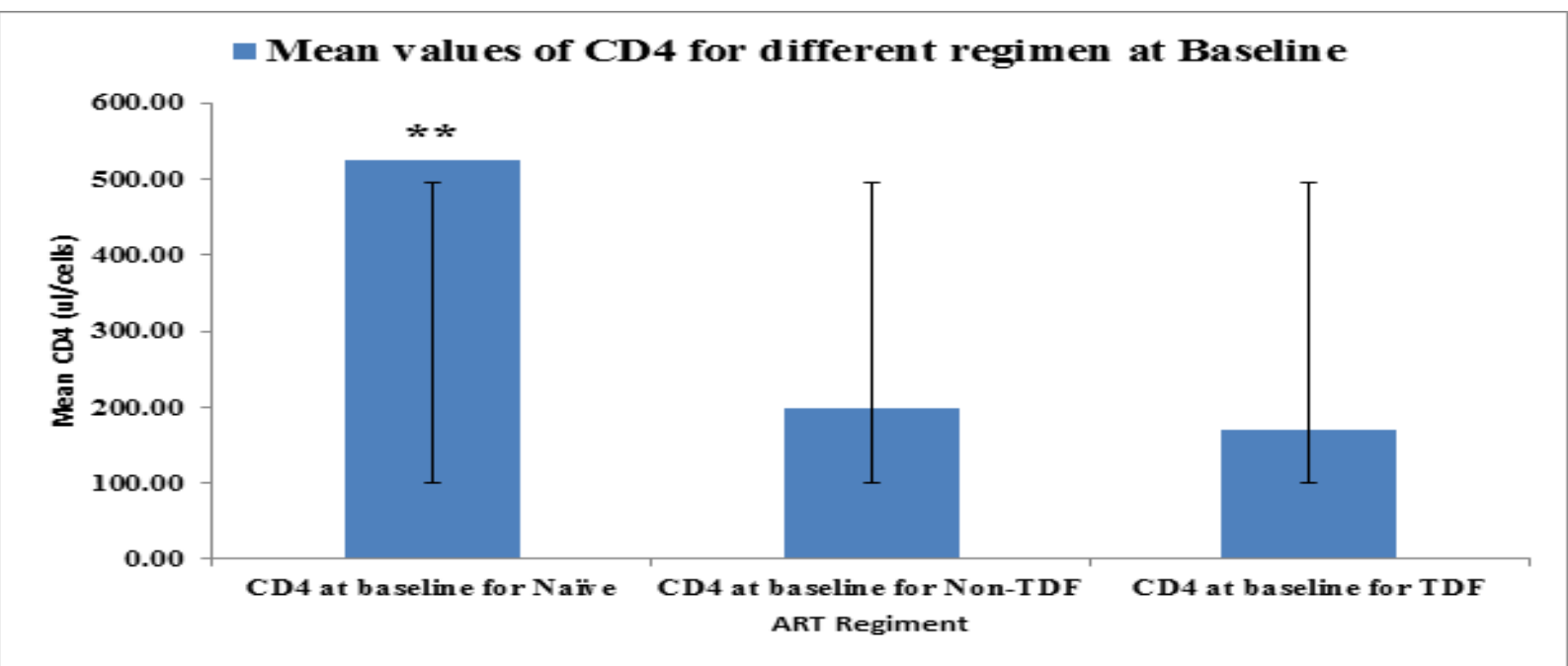

**: Significant at $\mathrm{p}=0.00$ and 0.00 when CD4at baseline for naïve was compared with CD4 at baseline for Non-TDF and TDF respectively'

Figure 1: CD4 of the study population at baseline 


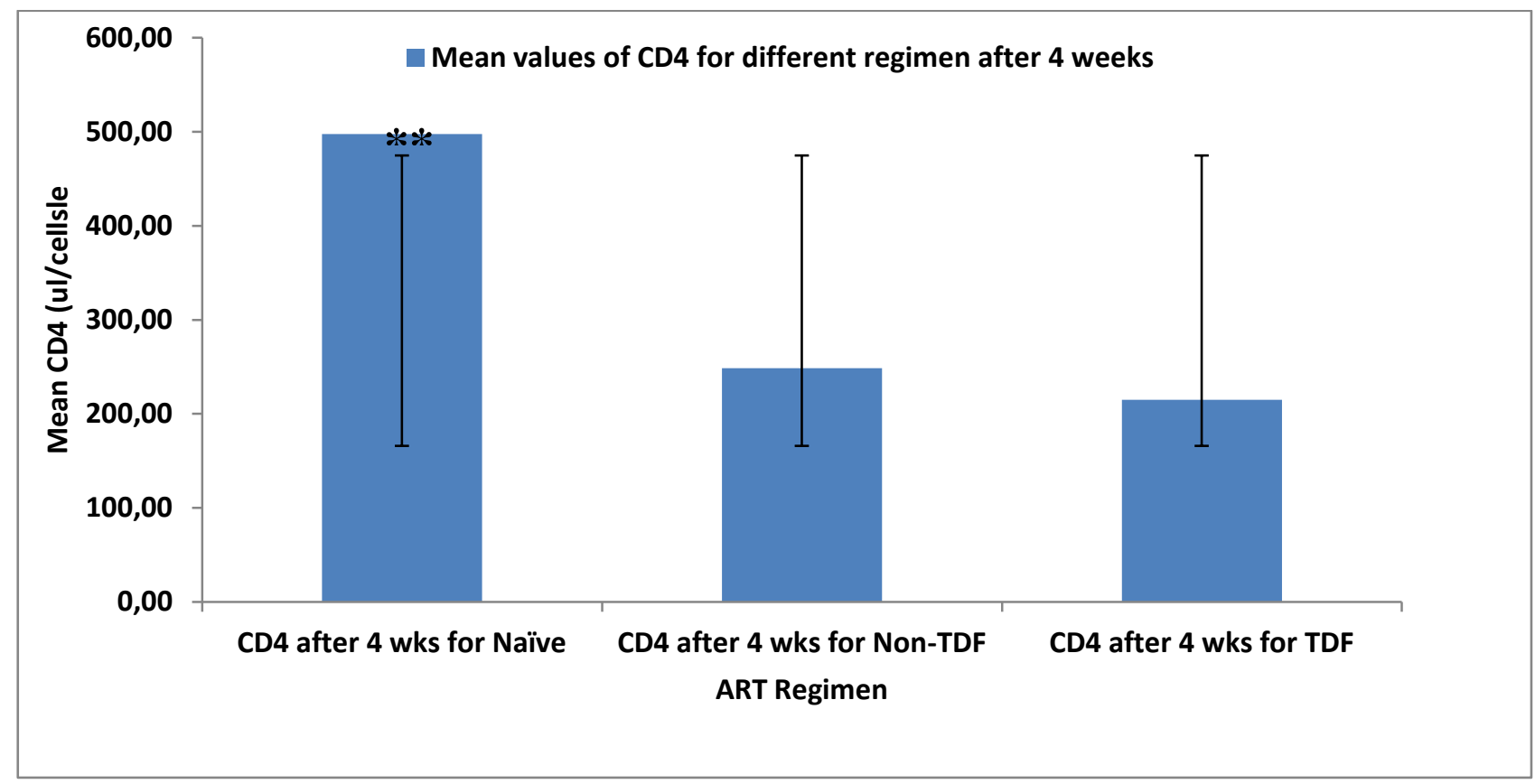

**: Significant at $\mathrm{p}=0.00$ and 0.00 when CD4after 4 wks for naïve was compared with CD4 after 4 wks for Non- TDF and TDF respectively

Figure 2: CD4 of the study population after 12 weeks of exposure

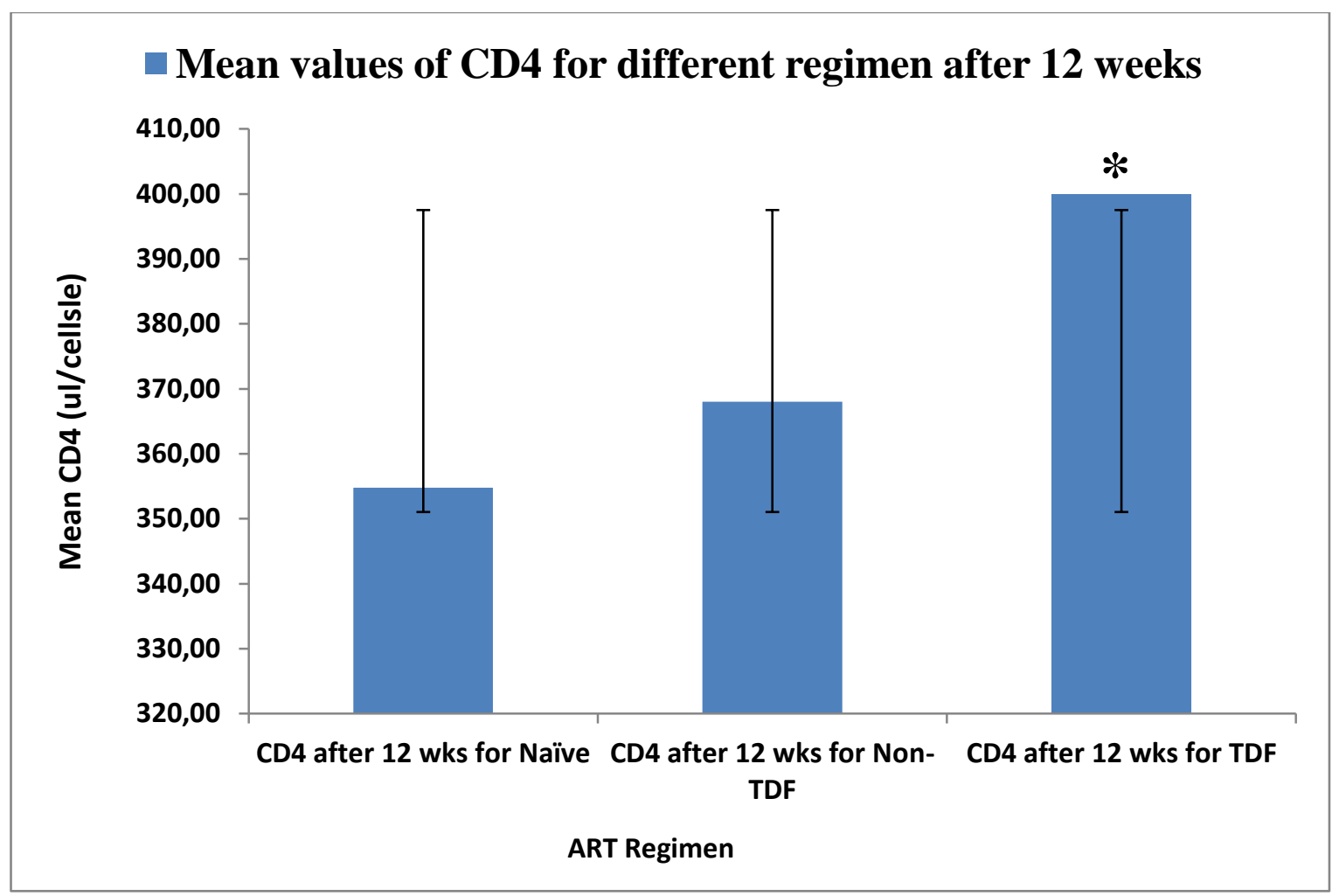

*: Significant at $\mathrm{p}=0.04$ when CD4after 12 wks for TDF was compared with CD4 after 12 wks for naïve.

Figure 3: CD4 of the study population after 12 weeks of exposure 


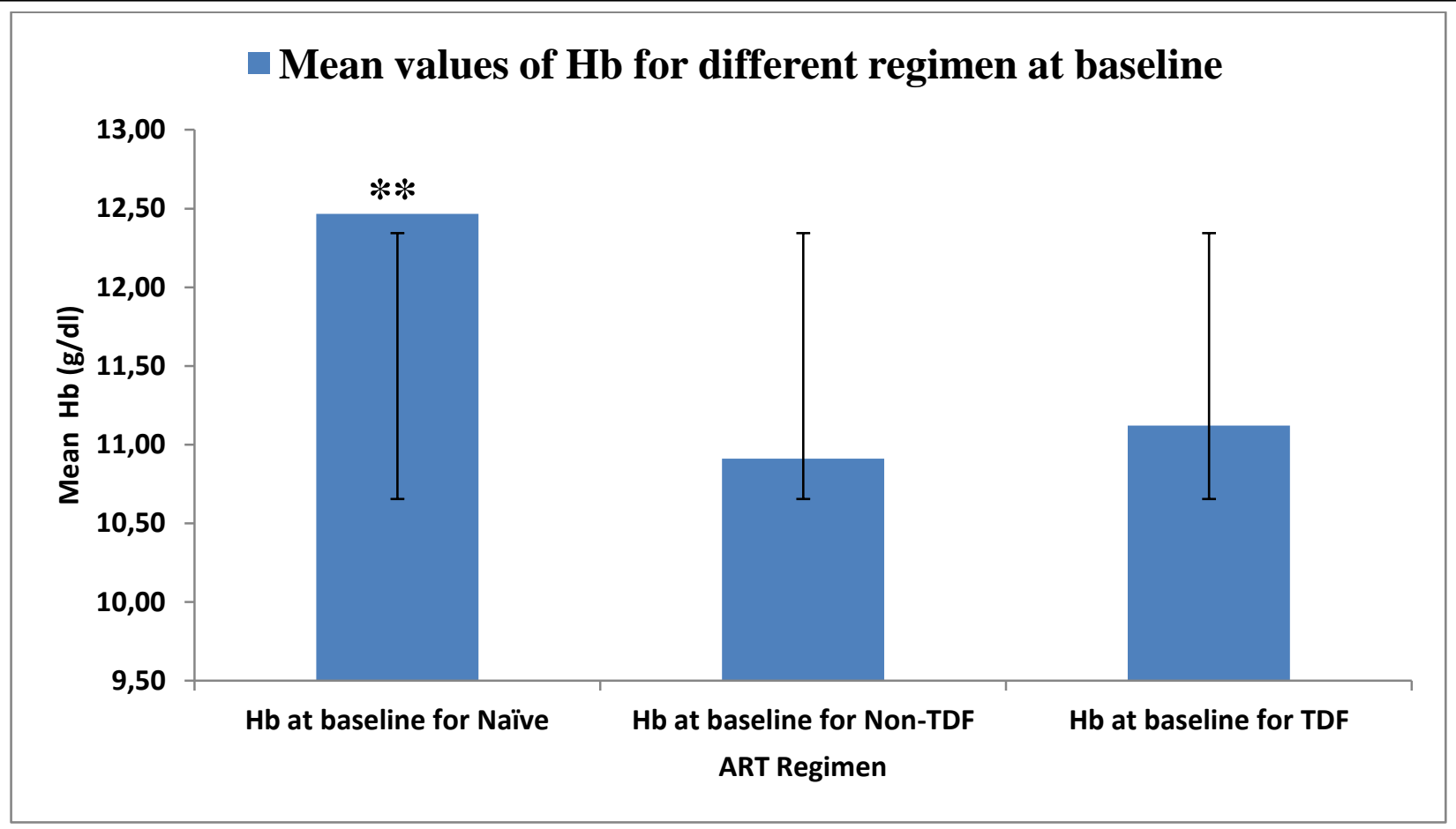

**: Significant at $\mathrm{p}=0.038$ and 0.016 when $\mathrm{Hbat}$ baseline for naïve was compared with $\mathrm{Hb}$ at baseline for Non-TDF and TDF respectively

Figure 4: $\mathrm{Hb}$ of the study population at baseline

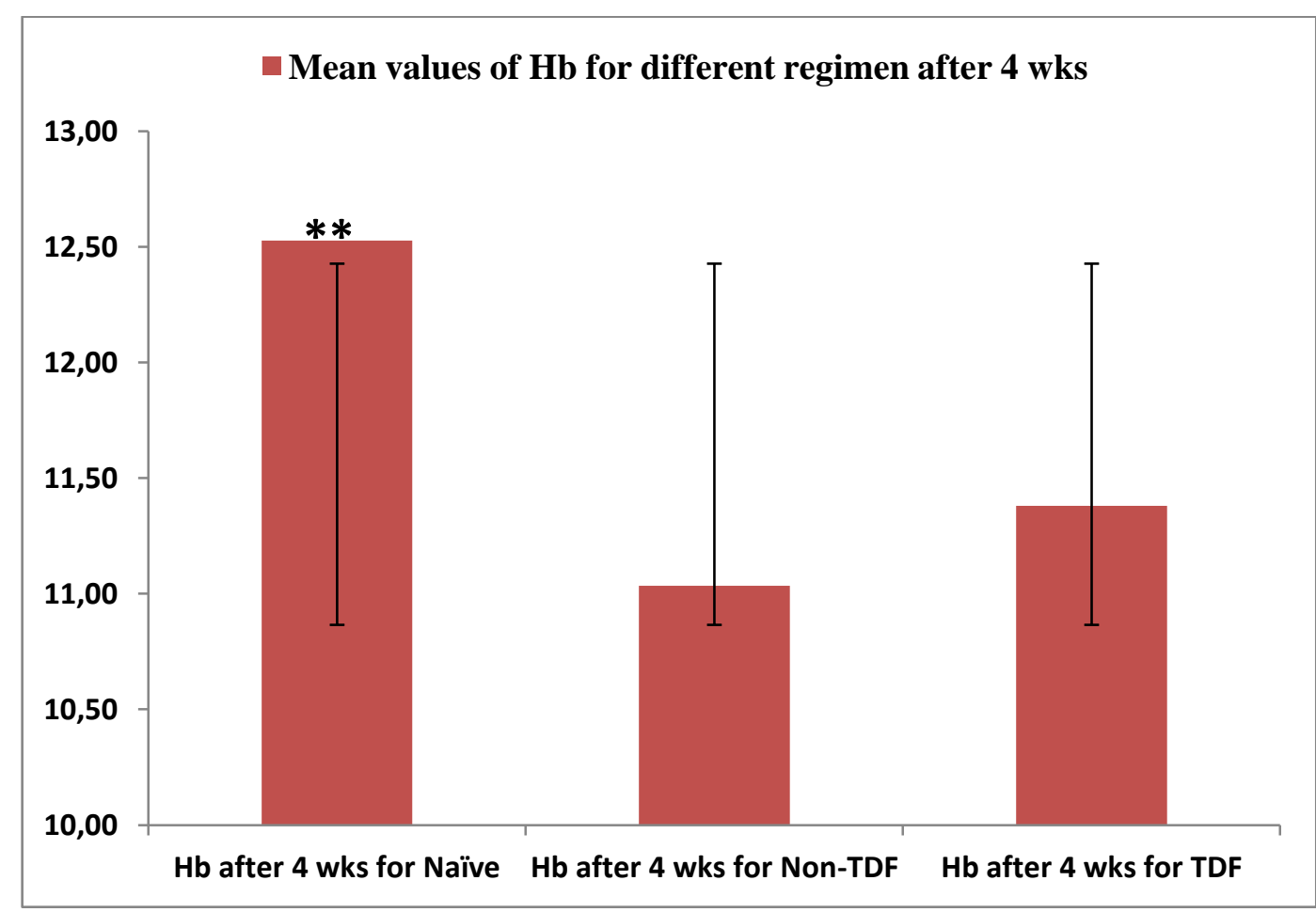

ART regimen

**: Significant at $\mathrm{p}=0.005$ and 0.008 when $\mathrm{Hb}$ after 4 wks for naïve was compared with $\mathrm{Hb}$ after 4 wks for Non-TDF and TDF respectively

Figure 5: $\mathrm{Hb}$ of the study population after 4 weeks of exposure 


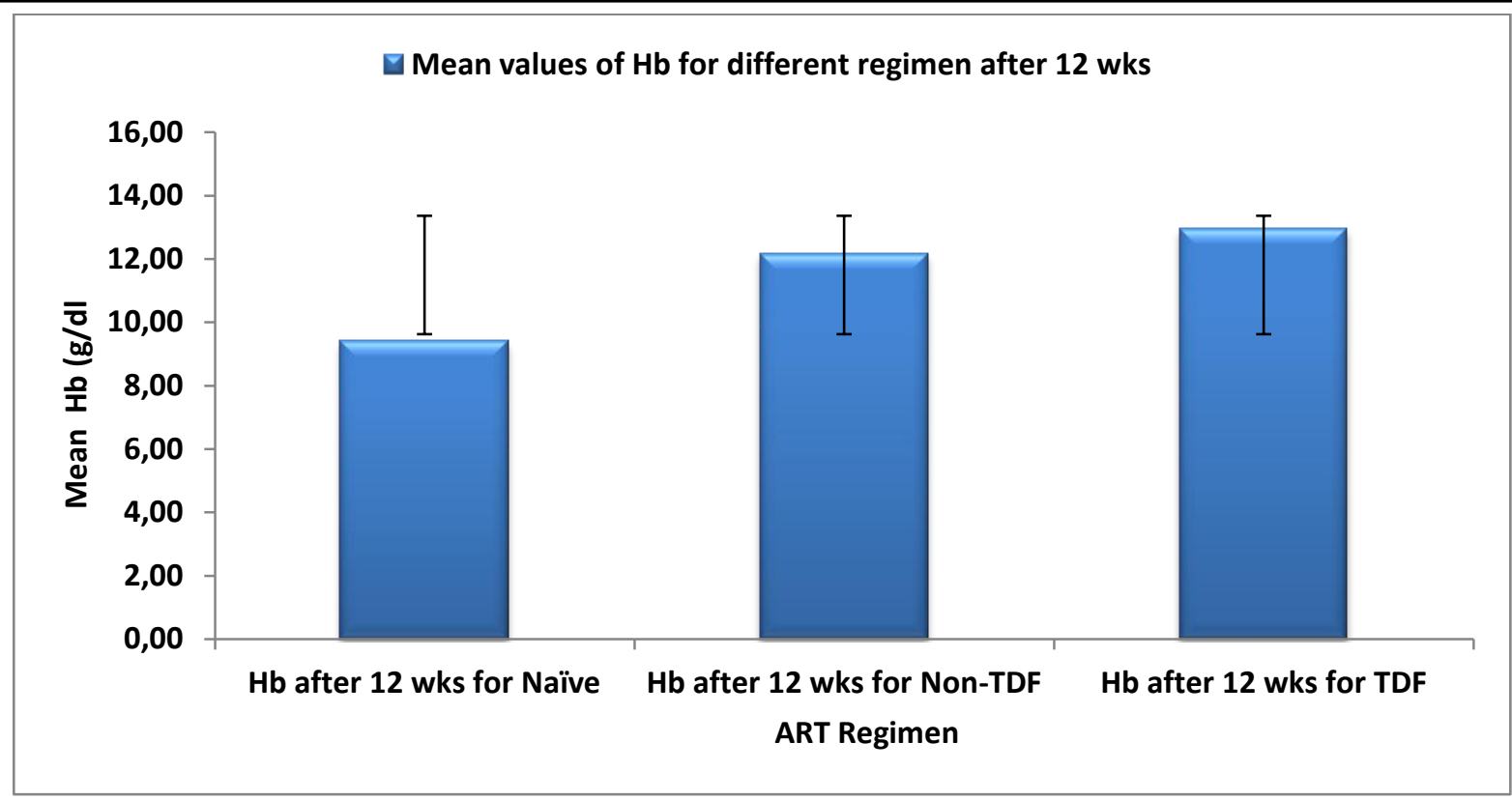

Figure 6: $\mathrm{Hb}$ of the study population after 12 weeks of exposure

At baseline, there was a statistical different between the haemoglobin level of the treatment Naïve group as compared to the TDF and NonTDF groups, this different was statistically significant $(\mathrm{p}=0.031$ and 0.016 respectively) (Figure 4). After 4 weeks there was a noticeable increase in all the groups and the different between the treatment-Naïve group and the TDF and Non-TDF groups was statistically significant ( $\mathrm{p}=0.005$ and 0.008 respectively) (Figure 5).

After 12 weeks, there was a decrease in the mean $\mathrm{Hb}$ values of the treatment-Naïve group while there was much higher increase in the TDF group as compared with the Non-TDF group (Figure 6).

\section{DISCUSSION}

In this longitudinal analysis of HIV-infected patients, we found that HIV-infected patients on TDF regimen had higher CD4 and Hb values than those on Non-TDF regimen. Studies has shown that in HIV-infected patients, it is important to control the viral load at a very lower level in order to maintain a positive CD4 count slope when ART continues at later stages of the infection, particularly from 3 months to more than 24 months after ART initiation. ${ }^{7}$.

This study agree with other findings ${ }^{8,}$ that HIVinfected patients have an initially delayed CD4 count recovery at 4 weeks after HAART initiation, but at 12 weeks, CD4 count increases tramendiously. This study examines the trend of CD4 count rather than the absolute CD4 count since ART initiation decreases CD4 count. We notice in particular that there is a progressive increased in CD4 of the TDF group after 12 weeks as compared to tenofovir sparing regimen groups (Non-TDF and treatment-naive). This finding is in consistent with other reports. ${ }^{9-11 .}$

TDF have been shown to boast the immune system in a non-specific way although a growing number of case reports and cohort studies have described an association between treatment with tenofovir and renal tubulopathy or impaired glomerular filtration rate (GFR) in patients with human immunodeficiency virus (HIV) infection. $^{12-14}$.

Haemoglobin level increased markedly in our series of patients especially after 4 weeks of exposure to antiretroviral therapy and in treatment Naïve patients while haemoglobin is increased markedly in the two drug treatment groups and decrease in the treatment Naïve group after 12 weeks of exposure. From our result it could be believe that tenofovir could be used in the treatment of HIV-infected Patients at risk of severe anemia as compared to the other antiretroviral therapy. 


\section{CONCLUSION}

Detecting the changes in CD4 and $\mathrm{Hb}$ for different treatment regimen is important in the HIVinfected population given its association with increased mortality risk and potential impact on treatment choices. In this longitudinal study of HIV-infected Naïve patients, TDF appeared more sensitive in detecting and boasting the immune system and causing lymphocyte proliferation than other antiretroviral therapy. Additionally, TDF might be used in patients at risk of anemia.

\section{COMPETING INTEREST}

The authors have declared that no competing interests exist

\section{REFERENCE}

1. MellorsJW, Munoz A, Giorgi JV.Plasma viral load and $\mathrm{CD}^{+}$lymphocytes as prognostic markers of HIV-1 infection. Ann Intern Med. 1997;126(12); 946-954.

2. Egger M, May T, Chene G. Prognosis of HIV-1-infected patients starting highly active antiretroviral therapy: a collaborative analysis of prospective studies. Lancet. 2002; 360(9327):119-129.

3. Martin J, Volberding P. (2010). HIV and premature aging: a field still in its infancy. Annals of Internal Medicine; 153: 477-9.

4. World Health Organization. World Health Report on HIV/AIDS 2004, 2004.

5. Mavigner $\mathrm{M}$, Delobel $\mathrm{P}$, Cazabat $\mathrm{M}$, Dubois M. HIV-1 Residual Viremia Correlates with persistent T-cell activation in poor immunological responders to combination Antiretroviral therapy. PLos ONE2009: $4(10)$. e7658.doi:10.1371/journal.pone.0007658

6. Kaufmann GR, Perrin L, Pantaleo G. CD4 $\mathrm{T}$ lymphocyte recovery in individuals with advance HIV-1 infection receiving potent ART for 4 years: the swiss HIV cohort study. Arch intern. Med. 2003;163 (18)2187-2195.
7. Konopnicki D, Mocroft A, de Wit S, Antunes F, Ledergerber B, Katlama C, Zilmer K, Vella S, Kirk O, Lundgren JD: Hepatitis B and HIV: prevalence, AIDS progression, response to highly active antiretroviral therapy and increased mortality in the EuroSIDA cohort. AIDS 2005, 19(6):593-601.

8. Law WP, Duncombe CJ, Mahanontharit A, Boyd MA, Ruxrungtham K, Lange JM, Phanuphak P, Cooper DA, Dore GJ: Impact of viral hepatitis co- infection on response to antiretroviral therapy and HIV disease progression in the HIV-NAT cohort. AIDS 2004, 18(8):1169-1177.

9. Akinola NO, Olasode O, Adediran IA, Onayemi $\mathrm{O}, \mathrm{Mu}$ - rainah $\mathrm{A}$, Irinoye $\mathrm{O}$, et al. The search for a predictor of CD4 cell count continues: total lymphocyte count is not a substi- tute for CD4 cell count in the management of HIV-infected individuals in a resource-limited setting. Clin Infect Dis 2004; 39(4): 579-81.

10. Spacek LA, Griswold M, Quinn TC, Moore RD. Total lym- phocyte count and hemoglobin combined in an algorithm to initiate the use of highly active antiretroviral therapy in re- source-limited settings. AIDS 2003; 17(9): 1311-7.

11. Badri M, Wood R. Usefulness of total lymphocyte count in monitoring highly active antiretroviral therapy in resourcelimited settings. AIDS 2003; 17(4): 541-5.

12. Barrios A, Garcia-Benayas T, GozalezLahoz J, Soriano V. Teno- fovir-related nephrotoxicity in HIV-infected patients. AIDS 2004; 18: 960-3.

13. Izzedine H, Harris M, Perazella MA. The nephrotoxic effects of HAART. Nat Rev Nephrol 2009; 5: 563-73.

14. Rodriguez-Novoa S, Labarga P, D'Avolio A, Impairment in kidney tubular function in patients receiving tenofovir is associated with higher tenofovir plasma concentrations. AIDS 2010; 24: 1064- 6 